\title{
Adsorption of Polyvinylpyrrolidone over the Silica Surface: As Affected by Pretreatment of Adsorbent and Molar Mass of Polymer Adsorbate
}

\author{
Laila M. Al-Harbi, ${ }^{1}$ Samia A. Kosa, ${ }^{1}$ Musa K. Baloch, ${ }^{2,3}$ Qaisar A. Bhatti, ${ }^{2}$ \\ and El-Sayed El-Badawey H. El-Mossalamy ${ }^{4}$ \\ ${ }^{1}$ Department of Chemistry, Faculty of Science, King Abdulaziz University, P.O. Box 80203, Jeddah 21589, Saudi Arabia \\ ${ }^{2}$ Institute of Chemical Sciences, Gomal University, Dera Ismail Khan 29050, Pakistan \\ ${ }^{3}$ Department of Chemistry, University of Sargodha, Subcampus Bhakkar, Bhakkar 30000, Pakistan \\ ${ }^{4}$ Department of Chemistry, Faculty of Science, Benha University, Benha 13518, Egypt \\ Correspondence should be addressed to Laila M. Al-Harbi; lalhrbi@kau.edu.sa
}

Received 31 October 2015; Revised 8 April 2016; Accepted 13 April 2016

Academic Editor: Angel Concheiro

Copyright ( 2016 Laila M. Al-Harbi et al. This is an open access article distributed under the Creative Commons Attribution License, which permits unrestricted use, distribution, and reproduction in any medium, provided the original work is properly cited.

The adsorption of polyvinylpyrrolidone over the surface of silica has been investigated. The impact of molar mass of the polymer, $\mathrm{pH}$, and pretreatment temperature of silica particles have been evaluated by means of FTIR spectroscopy and electrophoretic measurements. The silica particles used have narrow particle size distribution. The zeta potential of the aqueous silica suspension was decreased with the increase in $\mathrm{pH}$. The amount of polymer adsorbed was increased with the increase in pretreatment temperature, time, concentration, $\mathrm{pH}$, zeta potential, and molar mass of the polymer. The addition of polymer to the system increased the zeta potential due to adsorption of polymer on the surface of the particles. However, the impact increased with the increase in molecular mass of the polymer. The IR spectra obtained before and after adsorption of polymer concluded that, mostly, hydrogen bonding is responsible for the adsorption phenomena; however, hydrophobic interactions also play a significant role. The mechanism has been investigated and established through FTIR spectroscopy.

\section{Introduction}

Dispersion in both aqueous and nonaqueous media is used in numerous products including paints, dyestuffs, pigments, printing inks, papers, adhesives, cosmetics, detergents, ceramics, and pharmaceutical and pesticide formulations. Use of coagulants to clarify drinking water was practiced in ancient time in China and in Egypt. Mineral and environmental engineers have applied the concepts of colloid science to help them utilize and preserve enormous resources which otherwise were waste material [1]. Nowadays, ceramic products ranging from building bricks to expensive china to rocket parts are made from clay-water sols by applying principles of colloid science [2]. The wide use of colloid science in the areas of cosmetics and detergents has added many products to our daily life [3]. Knowledge of human life, either written on paper or stored on film or disk, has depended upon the application of colloidal science in papermaking and development of new magnetic and electronic materials, and so forth [4]. The dispersion activity of such material is mostly controlled through the addition of polymer or a mixture of polymers and surfactants and the interactions of surfactants and/or neutral water polymers in aqueous solutions with solid particles play a vital role in such applications. Therefore, the investigation of these interactions has been an active field of interest [5-9]. On the other hand, the interaction of polymers with solid/liquid interface has not attained so much attention, despite the importance of polymer adsorption over the solid/liquid interface [10-15]. Further, the degree and nature of adsorption of polymer depend on the suspended 
TABLE 1: Physicochemical properties of AEROSIL OX 50, as per the manufacturer information.

\begin{tabular}{lcccccc}
\hline Source & BET surface area & $\mathrm{PZC}$ at $\mathrm{pH}$ & $\mathrm{SiO}_{2 \text { Contents }}$ & $\mathrm{pH}$ & Primary particle size & Tapped approximate density \\
\hline Evonik Industries & $50+15\left(\mathrm{~m}^{2} \mathrm{~g}^{-1}\right)$ & 3.2 & $\geq 99.8$ & $3.8-4.8$ & $0.4(\mu \mathrm{m})$ & $.130(\mathrm{~g} / \mathrm{L})$ \\
\hline
\end{tabular}

"PZC at pH" signifies the PZC (point of zero charge) at $\mathrm{pH}$ at which surface charge of the particle is zero.

particles and hence affect the stability of the dispersion [16]. In addition to that, the surface charge-to-mass ratio of the particle plays a very important role in stabilizing the dispersion and this parameter is very much dependent upon the $\mathrm{pH}$ of the solution, size of particles, and so forth. Although a number of research papers have been published to understand the mechanism of adsorption of polymers over the solid surfaces, unfortunately, none of the papers deals with the prediction of adsorption behavior of a polymer over the solid surface up to the required level $[17,18]$.

Polyvinylpyrrolidone, also named Povidone or PVP, is a nonionic amphiphilic polymer. Because of its exceptional combination of properties like low toxicity, biocompatibility, film forming and adhesive characteristics, complexing ability to proton donors, and low osmotic pressure, it finds diverse applications in various fields of life like pharmaceutical and cosmetic products, food, adhesives, and textile auxiliaries as compared to other water-soluble polymers like polyethylene oxide and polyvinyl alcohol [19-21]. In spite of these facts, mechanisms involved in such processes are not well understood and hence need detailed and systematic exploration for proper utilization of silica $[6,12-14,22]$. AEROSIL ${ }^{\circledR}$ OX 50 because of its physicochemical properties has a large number of applications. Among fumed silica, AEROSIL OX 50, due to its specific properties, is widely used in biotechnology, agriculture, medicine, and pharmaceutics.

Since surface modification of an oxide via polymer adsorption leads to a change in its physicochemical characteristics, therefore, the main objective of this work was to investigate in detail the adsorption behavior of PVP onto silica in its aqueous suspension by using UV, FTIR spectroscopy, electrophoresis, and measurement techniques and to explore the impact of particle size of silica, temperature, pretreatment of adsorbent, molecular mass of polymer, and so forth on the adsorption phenomenon and to make use of that data for the exploration of the mechanism.

\section{Experimental}

2.1. Materials. AEROSIL OX 50, fumed silica oxide obtained from Degussa, Evonik Industries, Germany, was used as a model substrate. Some of the characteristics of AEROSIL OX 50 are presented in Table 1. These properties were also verified by employing a mercury porosity meter (Autosorb-1 der Firma Quanta chrome, USA), pH and LD measurements, and scanning electron microscopy. The specific surface area reported by the manufacturer was $50 \pm 15\left(\mathrm{~m}^{2} \cdot \mathrm{g}^{-1}\right)$ but when measured it was $40.72\left(\mathrm{~m}^{2} \cdot \mathrm{g}^{-1}\right)$.

Three different samples of PVP, also known as Luvitec, were obtained from BASF, Germany. PVP had three different molar masses and was used as a stabilizer for the silica dispersion. Some of the basic properties of PVP as provided
TABLE 2: Some of the basic properties of PVP as provided by the manufacturer, BASF, Germany.

\begin{tabular}{lccc}
\hline Sample & PVP K17 & PVP K30 & PVP K90 \\
\hline Mw/K dalton & $7-11$ & $45-55$ & $1200-2000$ \\
Degree of dispersity & $1.8 \pm 0.2$ & $2.3 \pm 0.2$ & $3.4 \pm 0.3$ \\
Rel. viscosity, 1\% in water & $1.075-1.110$ & $1.201-1.291$ & $3.991-6.197$ \\
\hline
\end{tabular}

by the manufacturer are listed in Table 2 . Water used for the dispersion was obtained from Millipore $\mathrm{GmbH}$, Schwalbach, Germany, having $\mathrm{pH}$ around 6.5 and conductance of $2.5 \mu \mathrm{S}$.

\subsection{Methods}

2.2.1. Characterization of Silica. The particle size and size distribution of silica were determined using scanning electron microscope (Jowl, Japan) and laser diffraction particle size analyzer Mastersizer 2000, Malvern Co., UK, equipped with impeller mixture. The surface area of the material was estimated using mercury porosity meter (Autosorb-1 der Firma Quanta chrome, USA). Surface charge was measured using Zetasizer 3000 (Malvern Co., Germany). The surface charge density $q$ in relation to $\mathrm{pH}$ was measured by polyelectrolyte titration using Particle Charge Detector (PCD, Mütek GmbH, Germany). FTIR Tensor, Bruker, Germany, was used for IR spectra measurement.

2.2.2. Pretreatment of Silica. For this purpose, the adsorbent (silica) was heated at five different temperatures from $298 \mathrm{~K}$ to $523 \mathrm{~K}$ for three hours in a vacuum oven (Gallon Kamp, UK) under inert atmosphere and then cooled to room temperature in a desiccant. The sample so obtained was used for adsorption studies [23].

2.2.3. Characterization of Polymer. The polymer was characterized by laser light scattering technique; the instrument used for the purpose was DAWN EOS, supplied by Wyatt, USA, with helium-neon laser of $632.8 \mathrm{~nm}$ wavelength as light source.

2.2.4. Adsorption Studies. Polymer solution was prepared in water having concentration $0-80 \mathrm{ppm}$. The solution was mixed with $0.05 \mathrm{~g}$ of preheated adsorbent $/ 20 \mathrm{~mL}$ of polymer solution in $100 \mathrm{~mL}$ sample bottles for 3 hours at different temperatures. The samples were equilibrated for 16 hours at $298 \mathrm{~K}$ at $85 \mathrm{rpm}$, using orbital shaker (Sanyo Orbit, Japan). Thereafter, the supernatant was removed from samples by centrifuging at $21000 \times \mathrm{g}$ for 15 minutes, using centrifuge model OSK12710 (Gawasieki Co., Japan), and analyzed for polymer concentration. 


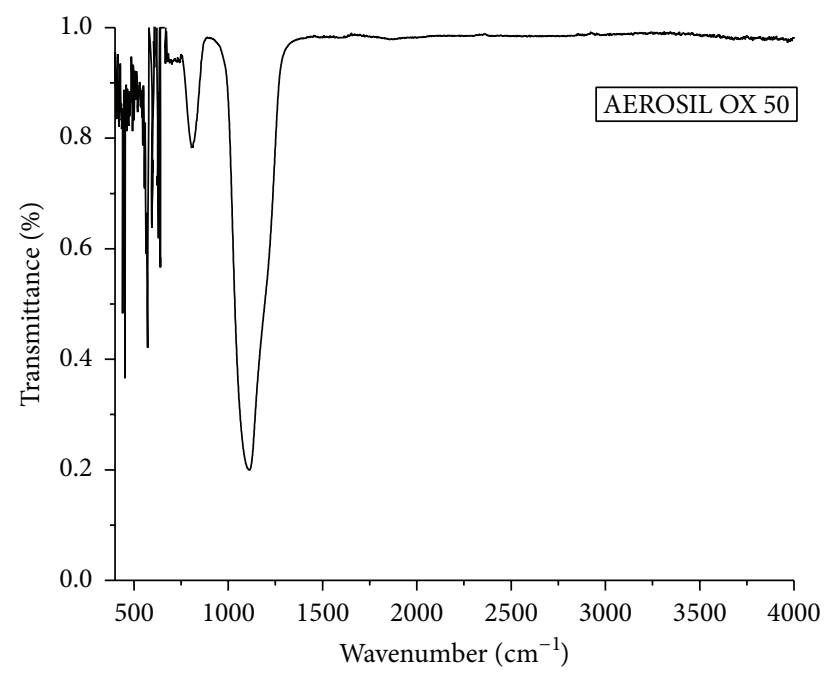

FIGURE 1: FTIR spectra of silica.

The $\mathrm{pH}$ dependent adsorption experiments were performed at $\mathrm{pH} 2-10$ at $298 \mathrm{~K}$. The $\mathrm{pH}$ of the solution was maintained using $\mathrm{HNO}_{3}$ and $\mathrm{KOH}$. pH of the solution was measured using $\mathrm{pH}$ meter supplied by Denwer, USA.

2.2.5. Analytical Measurements. The amount of unabsorbed PVP in the supernatant was determined using spectroscopic techniques. For this purpose, the UV-visible spectrophotometer employed was Lambda 800 (PerkinElmer) and $\lambda_{\max }$ used for measurement was $196 \mathrm{~nm}$.

2.2.6. Zeta Potential Measurement. The zeta potential of particles was measured using Zetasizer 3000, supplied by Malvern, Germany. $\zeta_{0}$ and $\zeta$ of silica were measured before and after the adsorption process. The contents of silica used during the electrophoretic measurements were $0.1 \mathrm{gL}^{-1}$. $\mathrm{KCl}$ was used as an electrolyte of $\left(1 \times 10^{-3} \mathrm{~mol} / \mathrm{L}\right)$ known concentration. Electrophoretic mobility of the samples was measured after allowing sufficient conditioning time to attain equilibrium at the required $\mathrm{pH}$ [24-26].

2.2.7. FTIR Spectroscopic Studies. FTIR spectra of silica, PVP, and PVP adsorbed over silica were recorded over the range of $4000 \mathrm{~cm}^{-1}$ to $400 \mathrm{~cm}^{-1}$ wavenumber by using $\mathrm{KBr}$ pellet (Figure 13). The instrument used for the purpose was FTIR Tensor, Bruker, Germany.

\section{Results and Discussion}

Fumed silica (AEROSIL OX 50) obtained from the supplier was characterized with respect to its purity, surface charge, size, and surface area. FTIR spectra of the samples are displayed in Figure 1.

The literature reveals that FTIR spectra of pure silica typically display peaks in two discrete regions, with wavenumbers greater than $2500 \mathrm{~cm}^{-1}$ and less than $1300 \mathrm{~cm}^{-1}[7,8]$. The former region corresponds to hydroxyl $(-\mathrm{OH})$ stretching due to absorbed or molecular water. However, in our case, we

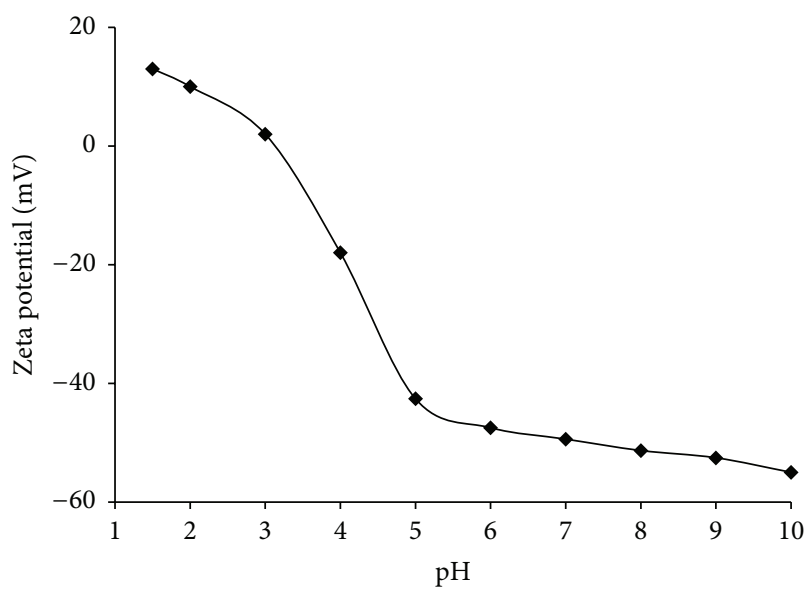

FIgURE 2: Zeta potential of $1 \%$ aqueous dispersion AEROSIL OX 50, measured in $1 \times 10^{-3} \mathrm{M} \mathrm{KCl}$. The error percentage was about 5.0.

did not observe any peak in this region. It was attributed to heat treatment of the silica before adsorption. The latter region is observed due to different modes of silica [8] and three prominent peaks were observed. The peak at $460 \mathrm{~cm}^{-1}$ was assigned to rocking motion of oxygen atoms bridging silicon atoms in siloxane bonds $(\mathrm{Si}-\mathrm{O}-\mathrm{Si})$. A broader peak at $810 \mathrm{~cm}^{-1}$ was assigned to $\mathrm{O}-\mathrm{Si}-\mathrm{O}$ vibration mode of $\mathrm{SiO}_{2}$ which was due to symmetric vibrations of silicon atoms in a siloxane bond [27]. A peak at $1105 \mathrm{~cm}^{-1}$ along with weak absorption at $960 \mathrm{~cm}^{-1}$ was the largest peak observed in a silica spectrum and was assigned to $\mathrm{Si}-\mathrm{O}-\mathrm{Si}$ and $\mathrm{Si}-\mathrm{O}$ stretching vibration modes of isolated $\mathrm{Si}-\mathrm{OH}$ groups. Similar bands were observed by Guzenko et al. [9]. The values were in good agreement with those reported in the literature [7$9,28,29]$ indicating that the material was pure and heating was performed properly.

As the stability of dispersion also depends upon the charge of the particle, the surface potential of the particles was determined as a function of $\mathrm{pH}$, which was adjusted using $1 \times$ $10^{-3} \mathrm{M} \mathrm{KOH}$ and $1 \times 10^{-3} \mathrm{M} \mathrm{HNO}_{3}$. The measurement was made in $1 \times 10^{-3} \mathrm{M} \mathrm{KCl}$ solution at $25^{\circ} \mathrm{C}$, using Malvern Zeta Master. The results obtained are displayed as a function of $\mathrm{pH}$ in Figure 2 which shows a negative zeta potential in the $\mathrm{pH}$ range from 3.2 to 10 . The isoelectric point was about 3.2. It indicated that the dispersion with $\mathrm{pH}$ near the isoelectric point (3.2) was unstable. If the charge is high, the particles will remain separated and dispersed and the suspension will be stable and vice versa. According to the results, the charge and hence the stability should be the highest in basic $\mathrm{pH}$ range because the zeta potential in this range is high.

In aqueous suspensions of silica particles, the surface silicon and oxygen bonds are unsatisfied and are neutralized by $\mathrm{OH}^{-}$and $\mathrm{H}^{+}$species. As a result, partial or total particle surface hydroxylation can result in the formation of silanol groups $\left[\mathrm{Si}(\mathrm{OH})_{n}\right]$ which dissociate in pure water through the following reactions:

$$
\begin{aligned}
\mathrm{SiOH}+\mathrm{OH}^{-} & \longrightarrow \mathrm{Si}-\mathrm{O}^{-}+\mathrm{H}_{2} \mathrm{O} \\
\mathrm{SiOH}+\mathrm{H}^{+} & \longrightarrow \mathrm{Si}-\mathrm{OH}^{+}+\mathrm{H}_{2} \mathrm{O}
\end{aligned}
$$




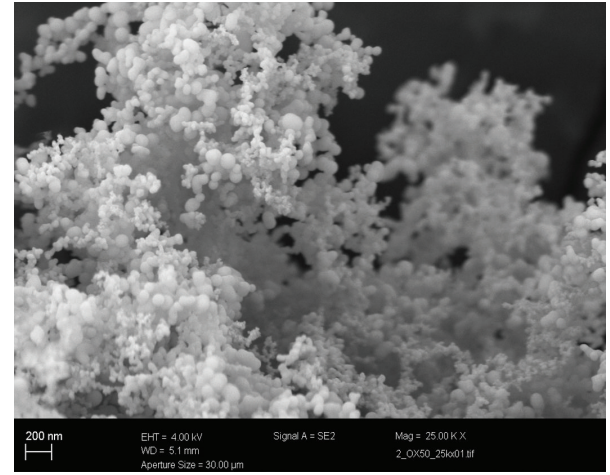

FIGURE 3: SEM picture of AEROSIL OX 50.

That is why silica particles may end negatively charged in water suspension for basic $\mathrm{pH}$ and positively charged under acidic $\mathrm{pH}[30,31]$. These phenomena impact directly the zeta potential behavior of silica suspensions and cause a slight change in zeta potential with a change in $\mathrm{pH}$.

The rest of the measurements were, therefore, performed at $\mathrm{pH} 6$ at room temperature with a negative zeta potential of about $-47 \mathrm{mV}$.

The electron micrographs of AEROSIL OX 50 are displayed in Figure 3. The micrographs showed that although the particles are monodispersed, they are aggregated which may affect their adsorption and dispersion and stabilization properties. Although ultrasonication was performed for $30 \mathrm{~min}$, it was impossible to break the aggregates completely as concluded by the manufacturer.

The results obtained from Mastersizer after homogenizing the system with magnetic stirrer for one hour and ultrasonication for various time periods are shown in Figure 4.

The figure indicates monodispersity in the size of the particles; however, some aggregates are visible in the images. The particle sizes obtained in the aqueous dispersed phase for the samples sonicated for different times ranged from 220 to $900 \mathrm{~nm}$ with an average of $400 \mathrm{~nm}$, as is displayed in Figure 4. These plots also show monodispersity to some extent while the polydispersity is attributed to aggregates that are present in the system. Furthermore, these observations are in accord with information provided by the manufacturer.

The figure also indicated that the sonication time has an almost negligible impact on the size and size distribution. However, the degree of dispersity was first decreased and then was increased if the sonication time was longer than $20 \mathrm{~min}$. This trend was attributed to the balance between shear and colloidal forces. This phenomenon is further supported by

$$
\gamma_{c}=f\left(\frac{s \sigma}{\eta_{A} a}\right) .
$$

Here, $\gamma_{c}$ is the critical shear rate, $s$ is the ratio of particle to medium viscosity, $\sigma$ is interfacial tension, $\eta_{A}$ is droplet viscosity, and $a$ is the size of the particle.

Equation (3) states that aggregate formation or disintegration depends upon the critical shear rate. It is important

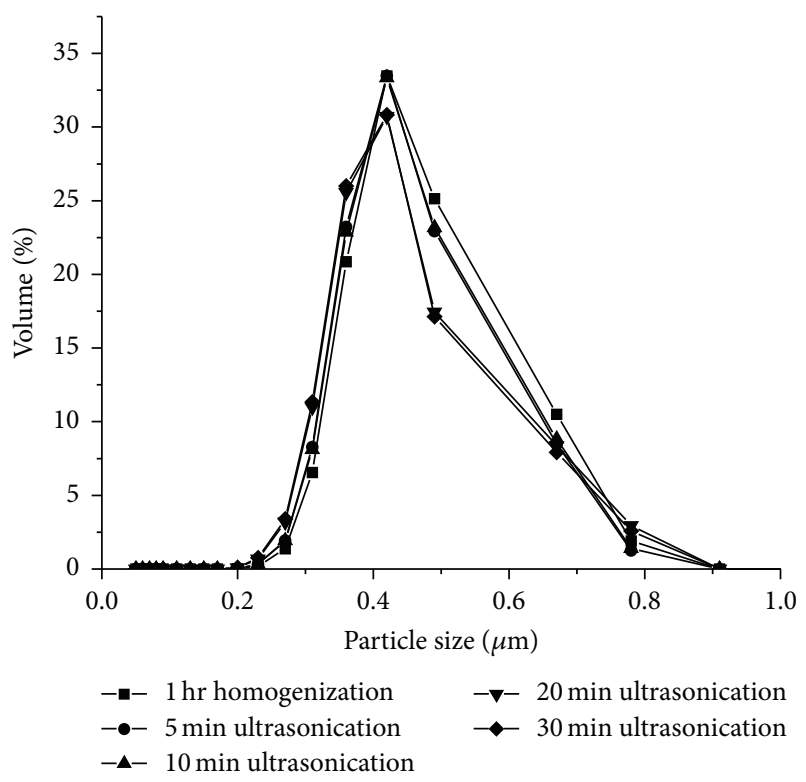

FIgURE 4: Particle size distribution of AEROSIL OX 50 (1\% aqueous dispersion) measured after sonification for various duration using Mastersizer. The error percentage was about 3.5\%.

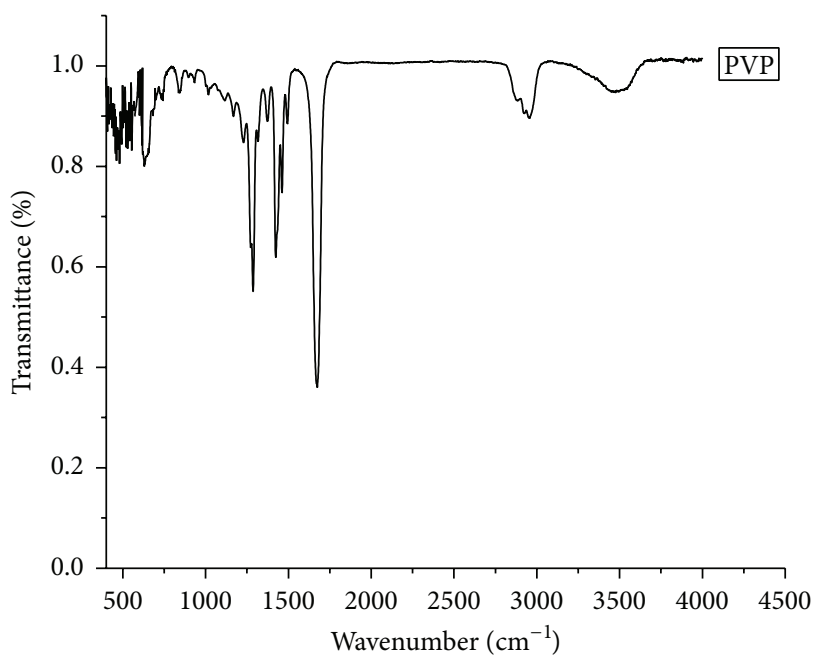

FIGURE 5: FTIR spectra of polyvinylpyrrolidone.

to note that the critical shear rate is inversely proportional to the size of the particles [32]. This means that if the size of the particles is small, then the high shear rate is needed to further decrease the size.

The FTIR spectra of the polymer (PVP) used showed a good agreement with the literature indicating that the polymer was pure (Figure 5) [10,29].

The results obtained for molecular weight and degree of dispersity of polymer were $9.18(1.8), 49.96(2.3)$, and $1.43 \times 10^{3}$ (3.4) $\mathrm{Kg} / \mathrm{mol}$. It was noted that with the increase in molecular mass of the polymer the degree of dispersity was increased and the results were the same (within experimental error) as supplied by the manufacturer shown in Table 2. 


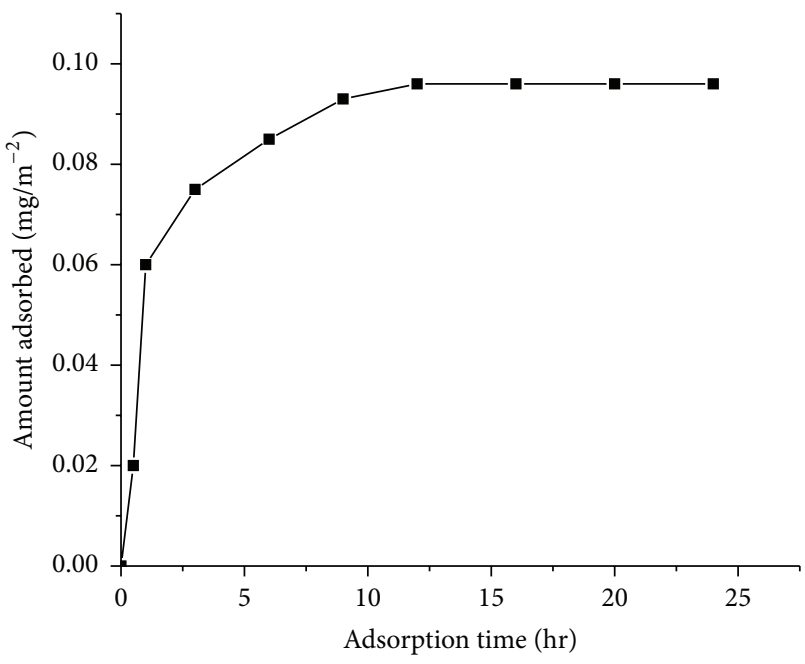

FIGURE 6: Adsorbed amount of PVP as a function of time. The error percentage was about 2.5 .

Time dependent adsorption curves are frequently observed in polymer adsorption [29]. It is sometimes assumed that reconfirmation processes play an important role in determining the rate of adsorption. However, for polydisperse polymers, this is not the only possibility since small chains on the surface may be replaced by the bigger ones, leading to a slow increase in adsorbed amount. Although the time scale of such processes in not known, it is expected that they contribute significantly to the overall time dependent adsorption. Therefore, the amount of K90 adsorbed was measured after 24 hours of mixing. The silica concentration was $0.5 \mathrm{~g} / \mathrm{dm}^{-3}$ and the starting concentration of the PVP was $80 \mathrm{ppm}$. It can be seen that the adsorbed amount was increased sharply during the first 10 hours and then leveled off (Figure 6). On the bases of these observations and for practical reasons, we chose 16 hours as adsorption time in all adsorption experiments.

Adsorption isotherms for the polymer of different molecular masses are shown in Figure 7. It was noted that the isotherms were of Langmuir type and of high affinity and that the amount adsorbed was increased from 0.4 to $0.8 \mathrm{mg} \mathrm{m}^{-2}$ with the increase in molecular mass of the polymer $[33,34]$. It was noted from the isotherms that initially the adsorption was increased very rapidly with the increase in concentration of polymer and ultimately it leveled off. This was accredited to the fact that initially the availability of the surface free sites was high and hence adsorption took place faster. However, as the time passed, the equilibrium was established and availability of free sites was reduced to almost zero and hence the adsorption became difficult and rate of adsorption became almost zero. The maximum amount of polymer adsorbed onto the surface of silica was increased with the concentration of polymer. These findings were in line with the expectations $[35,36]$.

The amount of polymer (PVP) adsorbed per unit surface area was calculated using Langmuir adsorption. The data gave exactly straight lines and the average value obtained in

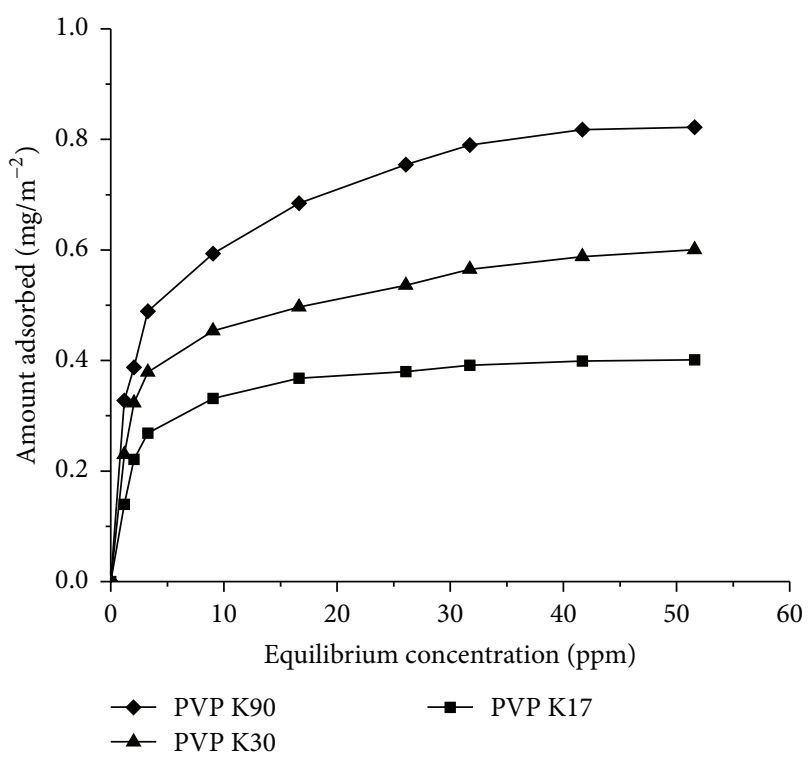

FIGURE 7: Adsorption isotherms of PVP having different molecular mass on AEROSIL OX 50, measured at $298 \mathrm{~K}$. The error percentage was about 2.5 .

this way was $0.6 \mathrm{mg} \cdot \mathrm{m}^{-2}$ (Figure 7). However, this value was slightly less than $\left(1.0 \mathrm{mg} \cdot \mathrm{m}^{-2}\right)$ the adsorbed concentration for a calculated condensed monolayer of monomer units.

Although the adsorbed amount was increased much with increasing the molar mass of the polymer, for higher molecular mass, leveling off of adsorption took place. The maximum amount adsorbed at plateau region was consistent with the data published in the literature $[37,38]$. The adsorption capacity was found to be a function of molecular mass, showing similar dependency to that reported by Cohen Stuart et al. [39]. Polymer-solvent interaction and segmental adsorption energy parameter are the two main factors which may work behind this phenomenon. Low polymer-solvent interaction is a poor solvent and high segmental adsorption energy parameter. That is, strong attachment of the segments may lead to a high adsorbed amount of polymer [39].

The amount adsorbed at plateau coverage was plotted as a function of $\log M w$ in Figure 8. It was observed that the increase in adsorption was very sharp for low molecular mass and ultimately leveled off for high molecular mass. Since we have used polymer with only three molecular masses and worked with lower concentrations, therefore, for a better understanding of this trend, data reported by Cohen Stuart et al. and Robinson and Williams [34, 38, 40] were also plotted in Figure 8 for comparison purpose and the results showed an excellent agreement. It is interesting to note that although the amount adsorbed was increased with the molecular weight and then leveled off, the values in $\mathrm{mM} / \mathrm{m}^{2}$ were decreased and followed a single exponential relationship irrespective of data source (Figure 8). This type of behavior could be explained in terms of variation in conformation of polymer chains with the molecular mass.

In order to find out the effect of $\mathrm{pH}$ over the adsorption of polymer, we measured the adsorbed amount at various $\mathrm{pH}$. 


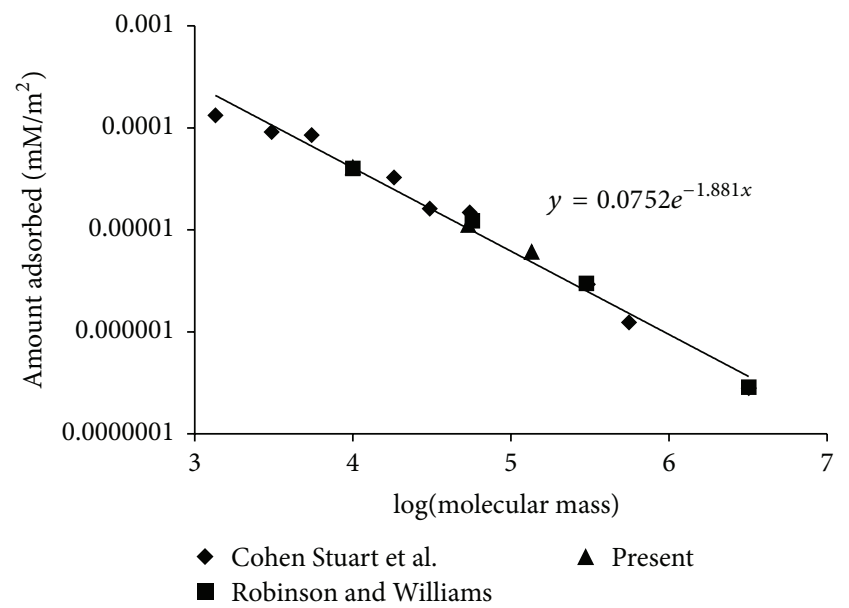

FIGURE 8: The amount of polymer adsorbed as a function of molecular mass of polymer.

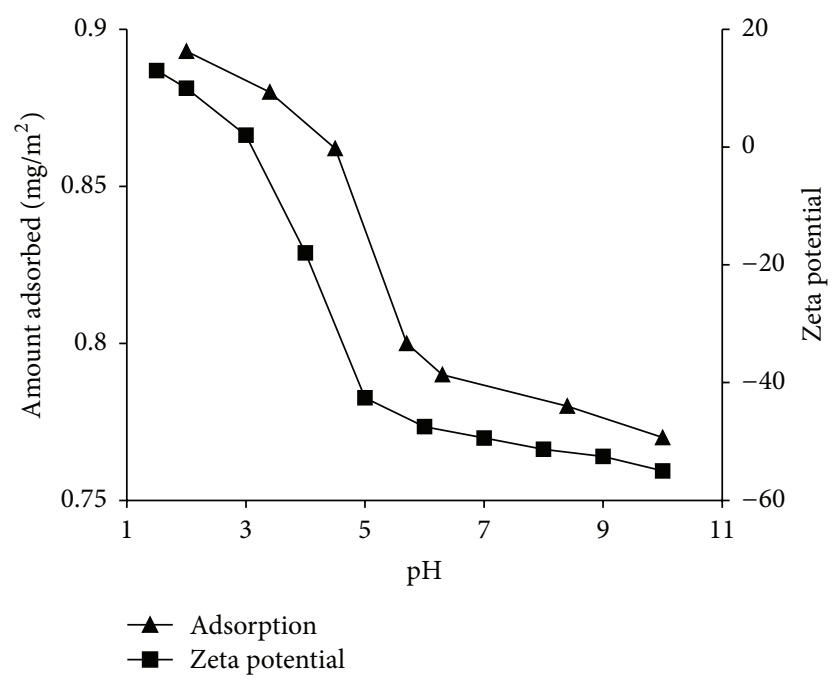

FIGURE 9: Amount of polymer adsorbed over silica as a function of $\mathrm{pH}$. The error percentage was about 2.5 in adsorption and in case of zeta potential it was $5 \%$.

The results obtained are plotted in Figure 9. The results concluded that the adsorbed amount is the highest at low $\mathrm{pH}$ and was decreased with the increase in $\mathrm{pH}$ and ultimately levels off. The same trend was observed for all the polymer samples indicating that the $\mathrm{pH}$ had the same impact irrespective of molecular mass. To explore the cause of such trend, the zeta potential and amount adsorbed have been plotted against $\mathrm{pH}$ in the same figure. The figure concludes that the adsorption is very much related to surface charge of the solid material used. Further to it, the solvent quality will deteriorate for polymer with the increase in $\mathrm{pH}$ and hence conformation of polymer will change which may result in a decrease in adsorption of polymer.

Since it is observed that heating causes a reduction in the silanol-group density on the surface of silica particles [41,42], it is important to have some knowledge about the importance of these groups in the phenomenon of polymer adsorption.

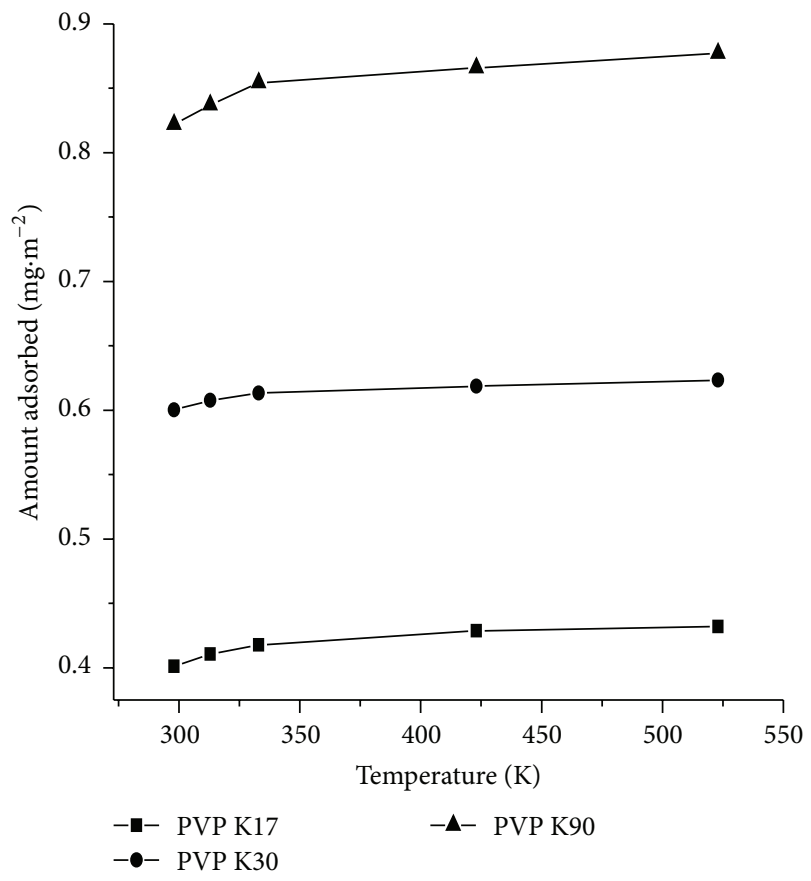

FIgURE 10: Effect of pretreatment of AEROSIL OX 50 on the adsorption of PVP. The error percentage was about 2.5.

Therefore, a study of the impact of preheating silica on the adsorption of PVP has been conducted. The adsorbent was heated for three hours from $298 \mathrm{~K}$ to $523 \mathrm{~K}$ at five different temperatures and used for adsorption purpose. The results obtained are plotted in Figure 10. The figure shows that preheating does not have a very pronounced impact on the adsorption for a specific temperature range. By increasing the pretreatment temperature, the amount of polymer adsorbed was increased sharply. Since we have worked over a range of $298 \mathrm{~K}$ to $523 \mathrm{~K}$, to get detailed information in this regard, we interpolated our data with that of Alagha et al. and Behrens et al. [43, 44]. It is observed that a very little impact is seen up to $523 \mathrm{~K}$, and at higher temperature of about $1273 \mathrm{~K}$ a sharp increase in amount adsorbed was observed (Figure 11).

The initial smaller increase in amount adsorbed is attributed to the fact that desorption of gases and/or water during heating of silica resulted in an increase in availability of free surface site for the adsorption and at the same time the decrease in surface area limits the amount adsorbed [4345]. Therefore, no significant amount adsorbed was observed. However, at higher temperature, a sharp increase revealed the fact that at higher temperature a significant reduction of surface area takes place along with the removal of surface silanol groups, which made the silica more hydrophobic, and an increase in the amount adsorbed is due to this hydrophobic interaction between polymer and the silica surface. Our findings are in very good agreement with those reported in the literature $[44,45]$.

The impact of preheating of silica on the adsorption of PVP was investigated by heating the samples at five different temperatures from $298 \mathrm{~K}$ to $523 \mathrm{~K}$ and the results obtained are plotted in Figure 11. The figure showed that 


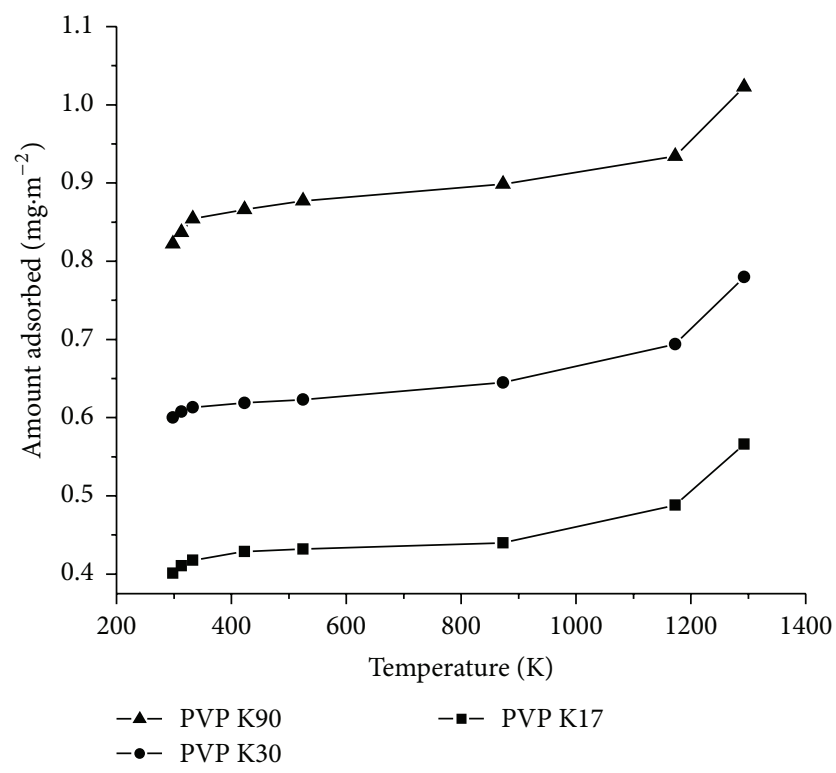

FIGURE 11: Effect of pretreatment of AEROSIL OX 50 on adsorption of PVP. The reported data are our own and the one reported by Cohen Stuart et al. [39].

by increasing the pretreatment temperature the amount of polymer adsorbed was increased significantly. An increase in the amount adsorbed for low temperature was attributed to desorption of gases and/or water during heating of silica, which resulted in an increase in availability of free surface site for the adsorption, and at the same time the decrease in surface area limited the amount adsorbed [41, 42]. Therefore, no significant amount was adsorbed. However, at high temperature, a significant increase in adsorption revealed that at higher temperature the removal of surface silanol groups took place $[42,43]$ which made the silica more hydrophobic and an increase in the amount adsorbed took place. However, a significant reduction of surface area also took place and that reduced the adsorption up to a considerable amount. Our findings are in very good agreement with those reported in the literature $[44,45]$.

Zeta potential of AEROSIL OX 50 after adsorption of PVP was obtained at $\mathrm{pH} 5.7$ and $25^{\circ} \mathrm{C}$. The results are displayed in Figure 11 as a function of PVP adsorbed amount. It was observed that the zeta potential of AEROSIL OX 50 was initially $-42.5 \mathrm{mV}$ and was significantly increased with the increase in concentration of PVP due to adsorption of polymer over AEROSIL OX 50. As $\mathrm{N}-\mathrm{C}=\mathrm{O}$ group is present in PVP and is electron donor in nature, it causes an increase in the surface charge on silica or enhances the shielding effect of the oxide surfaces [46] and hence was dependent upon concentration and molecular mass of PVP. This is the reason why the $\mathrm{pH}$ of the media was increased by the addition of the polymer. The figure also displayed that the charge over AEROSIL OX 50 was increased with the increase in molecular mass of PVP adsorbed. It was attributed to the shift of the share plan in case of adsorption of nonionic polymers [47] and the increase in number of monomers (active groups) in a chain of polymers.

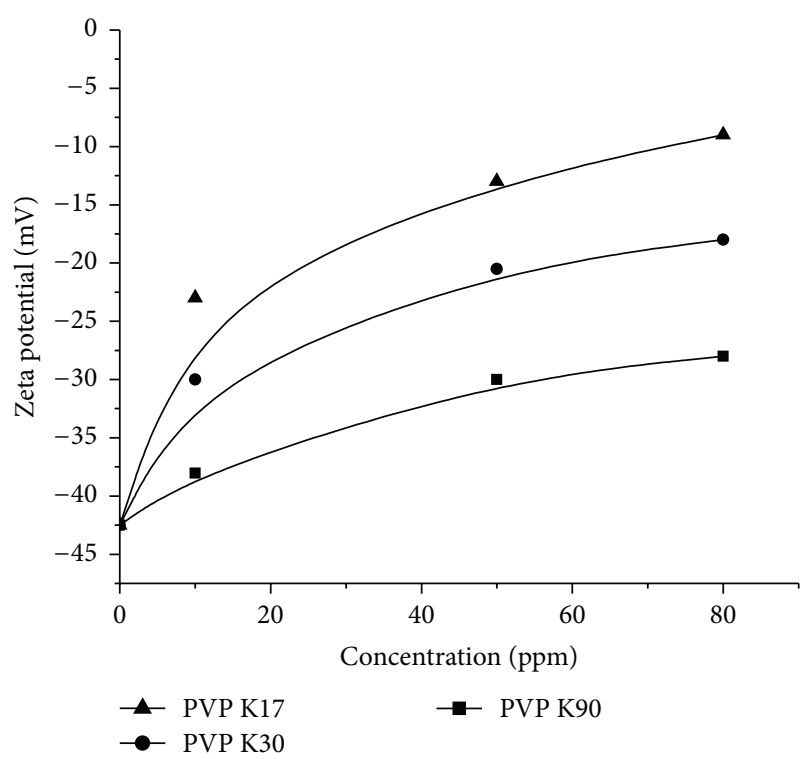

FIGURE 12: Effect of PVP concentration and molecular mass over the zeta potential of silica. The error percentage was about 5.0.

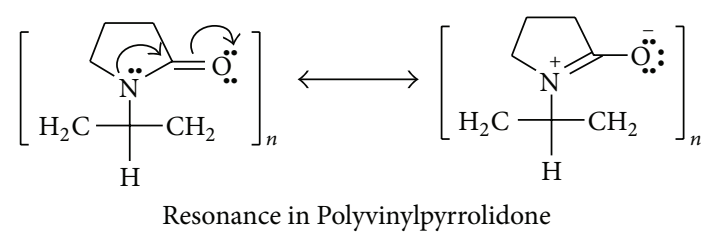

Scheme 1: Schematic presentation of resonating structure of polyvinylpyrrolidone.

To establish the mechanism of PVP adsorption onto AEROSIL OX 50, FTIR spectroscopic measurements of the samples were carried out before and after the adsorption of PVP. The spectra so recorded for AEROSIL OX 50, PVP, and PVP adsorbed over AEROSIL OX 50 are displayed in Figure 12 for comparison purpose. $\mathrm{CH}_{2}-$ band deformation near $1460 \mathrm{~cm}^{-1}$ in hydrocarbons and the splitting of the band into 1422,1460 , and $1493 \mathrm{~cm}^{-1}$ indicated the interchain forces in PVP and strain in the five-membered rings. Stretching of $\mathrm{CH}_{2}(\mathrm{CH}) \mathrm{NCO}$ in PVP was indicated by pronounced absorbance at $1287-1373 \mathrm{~cm}^{-1}$. The absorbance shown by carbonyl group in PVP at $1661 \mathrm{~cm}^{-1}$ was in the range reported earlier $[47,48]$. The presence of $\mathrm{C}=\mathrm{O}$ at $1661 \mathrm{~cm}^{-1}$ was significant, whereas the appearance of $\mathrm{C}-\mathrm{O}$ was very weak and may be due to the canonical forms of PVP as represented by the structure (Scheme 1).

Strong evidence which supported the presence of hydrogen bonding in adsorption of PVP on silica was that, before adsorption of PVP, the presence of $\mathrm{C}=\mathrm{O}$ at $1661 \mathrm{~cm}^{-1}$ was significant, whereas the appearance of $\mathrm{C}-\mathrm{O}$ was very weak; however, after the adsorption process, the $\mathrm{C}=\mathrm{O}$ group almost disappeared. Therefore, it was suggested that partial negative charged oxygen of the $\mathrm{C}=\mathrm{O}$ group caused the hydrogen bonding with the hydrogen of silanol. This was further supported by the fact that $\mathrm{PVP}$ has $\mathrm{N}-\mathrm{C}=\mathrm{O}$ groups which are 


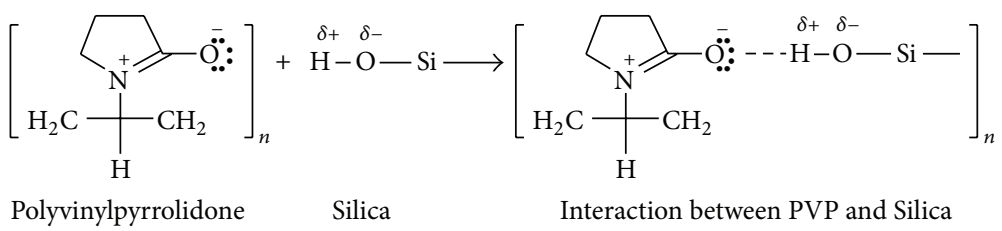

Scheme 2: Schematic presentation of the adsorption of PVP on silica.

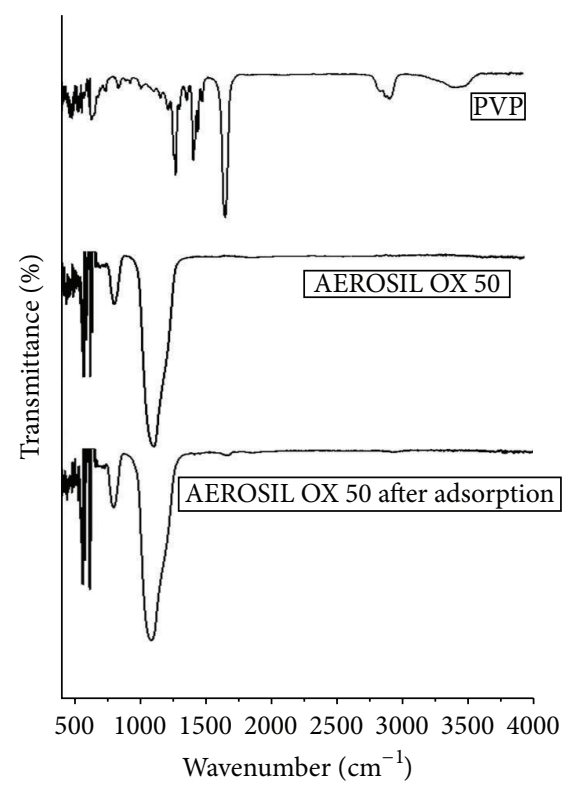

FIGURE 13: FTIR spectra of silica, PVP, and silica after adsorption of PVP.

electron donor in nature [49]; therefore, hydrogen bonding between oxygen of $\mathrm{C}=\mathrm{O}$ and hydrogen of silanols may have possibly taken place. Furthermore, there is no evidence for the conversion of $\mathrm{C}-\mathrm{N}$ into $\mathrm{C}=\mathrm{N}$ as shown by the shifting of peaks from $1661 \mathrm{~cm}^{-1}$ to $1078 \mathrm{~cm}^{-1}$ [50]. Schematic presentation of the mechanism of PVP adsorption on silica is given by Scheme 2 .

\section{Competing Interests}

The authors declare that they have no competing interests.

\section{Acknowledgments}

This work was funded by the Deanship of Scientific Research (DSR), King Abdulaziz University, Jeddah, under Grant no. 130-128-D1434. The authors, therefore, acknowledge with thanks the DSR for technical and financial support.

\section{References}

[1] P. Somasundaran, Y. H. C. Wang, and S. Acar, Future Trends in Polymer Science and Technology, Technomic Publishing, Berlin, Germany, 1987.
[2] A. G. Evans and T. G. Langdon, "Structural material," Progress in Materials Science, vol. 21, p. 174, 1976.

[3] H. M. Remz, “Cosmet," Toiletries, vol. 103, p. 70, 1988.

[4] V. Novotny, "Applications of nonaqueous colloids," Colloids and Surfaces, vol. 24, no. 4, pp. 361-375, 1987.

[5] B. K. G. Theng, "Interactions of clay minerals with organic polymers. Some practical applications," Clays and Clay Minerals, vol. 18 , no. 6, pp. 357-362, 1970.

[6] C. Ma and C. Li, "Interaction between polyvinylpyrrolidone and sodium dodecyl sulfate at solid/liquid interface," Journal of Colloid and Interface Science, vol. 131, no. 2, pp. 485-492, 1989.

[7] G. M. Filippelli, J. W. Carnahan, D. G. E. A. Swann, and S. V. Patwardhan, "Application of Fourier Transform Infrared Spectroscopy (FTIR) for assessing biogenic silica sample purity in geochemical analyses and palaeoenvironmental research," Climate of the Past, vol. 7, no. 1, pp. 65-74, 2011.

[8] M. Waseem, S. Mustafa, A. Naeem, and K. H. Shah, "Irfan shah and ihsan-ul-haque," Pakistan Material Society, vol. 3, no. 1, 2009.

[9] N. V. Guzenko, E. M. Pakhlov, N. A. Lipkovskaya, and E. F. Voronin, "Sorption modification of fine silica with polyvinylpyrrolidone," Russian Journal of Applied Chemistry, vol. 74, no. 12, pp. 2017-2020, 2001.

[10] S. Robinson and P. A. Williams, "Inhibition of protein adsorption onto silica by polyvinylpyrrolidone," Langmuir, vol. 18, no. 23, pp. 8743-8748, 2002.

[11] Th. F. Tadros, "The interaction of cetyltrimethylammonium bromide and sodium dodecylbenzene sulfonate with polyvinyl alcohol. adsorption of the polymer-surfactant complexes on silica," Journal of Colloid and Interface Science, vol. 46, no. 3, pp. 528-540, 1974.

[12] K. Esumi and M. Oyama, "Simultaneous adsorption of poly(vinylpyrrolidone) and cationic surfactant from their mixed solutions on silica," Langmuir, vol. 9, no. 8, pp. 20202023, 1993.

[13] K. Esumi and Y. Yamanaka, "Interaction between sodium dodecyl poly(oxyethylene) sulfate and alumina surface in aqueous solution," Journal of Colloid and Interface Science, vol. 172, no. 1, pp. 116-120, 1995.

[14] B. M. Moudgil, S. Behl, and N. S. Kulkarni, "Measurement of heat of adsorption of polyethylene oxide on dolomite, silica, and alumina by microcalorimetry," Journal of Colloid And Interface Science, vol. 148, no. 2, pp. 337-342, 1992.

[15] K. Chari and W. C. Lenhart, "Effect of polyvinylpyrrolidone on the self-assembly of model hydrocarbon amphiphiles," Journal of Colloid and Interface Science, vol. 137, no. 1, pp. 204-216, 1990.

[16] A. H. Otsuka and K. Esumi, "Simultaneous adsorption of poly(vinylpyrrolidone) and anionic hydrocarbon/fluorocarbon surfactant from their binary mixtures on alumina," Langmuir, vol. 10, no. 1, pp. 45-50, 1994. 
[17] R. Bury, B. Desmazières, and C. Treiner, "Interactions between poly(vinylpyrrolidone) and ionic surfactants at various solid/water interfaces: a calorimetric investigation," Colloids and Surfaces A: Physicochemical and Engineering Aspects, vol. 127, no. 1-3, pp. 113-124, 1997.

[18] G. D. Parfitt, "The role of the surface in the dispersion of pmders in liquids," Pure and Applied Chemistry, vol. 53, pp. 2233-2240, 1991.

[19] V. E. Killmann and H. G. Wiegand, "Charakterisierung adsorbierter makromolekülschichten durch ellipsometrie," Die Makromolekulare Chemie, vol. 132, no. 1, pp. 239-258, 1970.

[20] C. W. Francis, "Adsorption of polyvinylpyrrolidone on reference clay minerals," Soil Science, vol. 115, no. 1, pp. 40-54, 1973.

[21] M. A. Cohen Stuart, G. J. Fleer, and B. H. Bijsterbosch, "The adsorption of poly(vinyl pyrrolidone) onto silica. I. Adsorbed amount," Journal of Colloid And Interface Science, vol. 90, no. 2, pp. 310-320, 1982.

[22] A. Fidalgo and L. M. Ilharco, "The defect structure of solgel-derived silica/polytetrahydrofuran hybrid films by FTIR," Journal of Non-Crystalline Solids, vol. 283, no. 1-3, pp. 144-154, 2001.

[23] Q. A. Bhatti, M. K. Baloch, S. Schwarz, and G. Petzold, "Impact of various parameters over the adsorption of polyvinylpyrrolidone onto kaolin," Journal of Dispersion Science and Technology, vol. 33, no. 12, pp. 1739-1745, 2012.

[24] J. Kim and D. F. Lawler, "Characteristics of zeta potential distribution in silica particles," Bulletin of the Korean Chemical Society, vol. 26, no. 7, pp. 1083-1089, 2005.

[25] J. A. A. Júnior and J. Baptista Baldo, "The behavior of zeta potential of silica suspensions," New Journal of Glass and Ceramics, vol. 4, no. 2, pp. 29-37, 2014.

[26] Q. A. Bhatti, M. K. Baloch, S. Schwarz, and G. Petzold, "Effect of various parameters on the stability of silica dispersions," Journal of Solution Chemistry, vol. 43, no. 11, pp. 1916-1928, 2014.

[27] Z. H. Cheng, A. Yasukawa, K. Kandori, and T. Ishikawa, "FTIR study on incorporation of $\mathrm{CO}_{2}$ into calcium hydroxyapatite," Journal of the Chemical Society, Faraday Transactions, vol. 94, no. 10, pp. 1501-1505, 1998.

[28] E. F. Voronin, L. V. Nosach, N. V. Guzenko, E. M. Pakhlov, and O. L. Gabchak, "Adsorption modification of nanosilica with non-volatile organic compounds in fluidized state," Nanomaterials and Supramolecular Structures: Physics, Chemistry, and Applications, pp. 169-177, 2010.

[29] R. Levy and C. W. Francis, "Interlayer adsorption of polyvinylpyrrolidone on montmorillonite," Journal of Colloid and Interface Science, vol. 50, no. 3, pp. 442-450, 1975.

[30] D. Frenkel, "Playing tricks with designer 'atoms," Science, vol. 296, no. 5565, pp. 65-66, 2002.

[31] P. M. Dove and J. D. Rimstidt, "Silica water interactions-in silica reviews in mineralogy," Mineral Society of America, vol. 29, pp. 259-307, 1994.

[32] G. I. Taylor, "The formation of emulsions in definable fields of flow," Proceedings of the Royal Society A, vol. 146, no. 858, pp. 501-523, 1934.

[33] A. Silberberg, "The adsorption of flexible macromolecules. Part I. The isolated macromolecule at a plane interface," The Journal of Physical Chemistry, vol. 66, no. 10, pp. 1872-1883, 1962.

[34] J. M. H. M. Scheutjens and G. J. Fleer, "Statistical theory of the adsorption of interacting chain molecules. 1. Partition function, segment density distribution, and adsorption isotherms," The Journal of Physical Chemistry, vol. 83, no. 12, pp. 1619-1635, 1979.
[35] M. A. Cohen Stuart, G. J. Fleer, and B. H. Bijsterbosch, "Adsorption of poly(vinyl pyrrolidone) on silica. II. The fraction of bound segments, measured by a variety of techniques," Journal of Colloid and Interface Science, vol. 90, no. 2, pp. 321334, 1982.

[36] B. K. G. Theng, "Clay-polymer interactions: summary and perspectives," Clay and Clay Minerals, vol. 30, no. 1, pp. 1-10, 1982.

[37] R. I. S. Gill and T. M. Herrington, “The flocculation of kaolin suspensions with cationic polyacrylamides of varying molar mass but the same cationic character," Colloids and Surfaces, vol. 22, no. 1, pp. 51-76, 1987.

[38] R. I. S. Gill and T. M. Herrington, "The effect of surface charge on the flocculation of kaolin suspensions with cationic polyacrylamides of varying molar mass but similar cationic character," Colloids and Surfaces, vol. 25, no. 2-4, pp. 297-310, 1987.

[39] M. A. Cohen Stuart, J. M. H. M. Scheutjens, and G. J. Fleer, "Polydispersity effects and the interpretation of polymer adsorption isotherms," Journal of Polymer Science: Polymer Physics Edition, vol. 18, pp. 559-573, 1980.

[40] K.-Ch. Ullman, Stabilization of $\mathrm{TiO}_{2}$ dispersion with non-ionic water-soluble molecules [Ph.D. thesis], University of Stuttgart, 1989.

[41] H. D. Ackler, R. H. French, and Y.-M. Chiang, "Comparisons of Hamaker constants for ceramic systems with intervening vacuum or water: from force laws and physical properties," Journal of Colloid and Interface Science, vol. 179, no. 2, pp. 460469, 1996.

[42] N. K. Adam, The Physics and Chemistry of Surfaces, Dover, New York, NY, USA, 1968.

[43] L. Alagha, S. Wang, L. Yan, Z. Xu, and J. Masliyah, "Spectroscopic investigation of the adsorption mechanisms of polyacrylamide polymers onto iron oxide particles," Langmuir, vol. 29, no. 12, pp. 3989-3998, 2013.

[44] S. H. Behrens, M. Borkovec, and P. Schurtenberger, "Aggregation in charge-stabilized colloidal suspensions revisited," Langmuir, vol. 14, no. 8, pp. 1951-1954, 1998.

[45] C. Friedsam, A. Del Campo Bécares, U. Jonas, M. Seitz, and H. E. Gaub, "Adsorption of polyacrylic acid on selfassembled monolayers investigated by single-molecule force spectroscopy," The New Journal of Physics, vol. 6, pp. 9-16, 2004.

[46] D. Grasso, K. Subramaniam, M. Butkus, K. Strevett, and J. Bergendahl, "A review of non-DLVO interactions in environmental colloidal systems," Reviews in Environmental Science and Biotechnology, vol. 1, no. 1, pp. 17-38, 2002.

[47] B. E. Novich and T. A. Ring, "Colloid stability of clays using photon correlation spectroscopy," Clays \& Clay Minerals, vol. 32, no. 5, pp. 400-406, 1984.

[48] M. Linsenbühler, J. H. Werth, S. M. Dammer et al., "Cluster size distribution of charged nanopowders in suspensions," Powder Technology, vol. 167, no. 3, pp. 124-133, 2006.

[49] L. Ghimici, S. Morariu, and M. Nichifor, "Separation of clay suspension by ionic dextran derivatives," Separation and Purification Technology, vol. 68, no. 2, pp. 165-171, 2009.

[50] R. S. Faibish, M. Elimelech, and Y. Cohen, "Effect of interparticle electrostatic double layer interactions on permeate flux decline in crossflow membrane filtration of colloidal suspensions: an experimental investigation," Journal of Colloid and Interface Science, vol. 204, no. 1, pp. 77-86, 1998. 

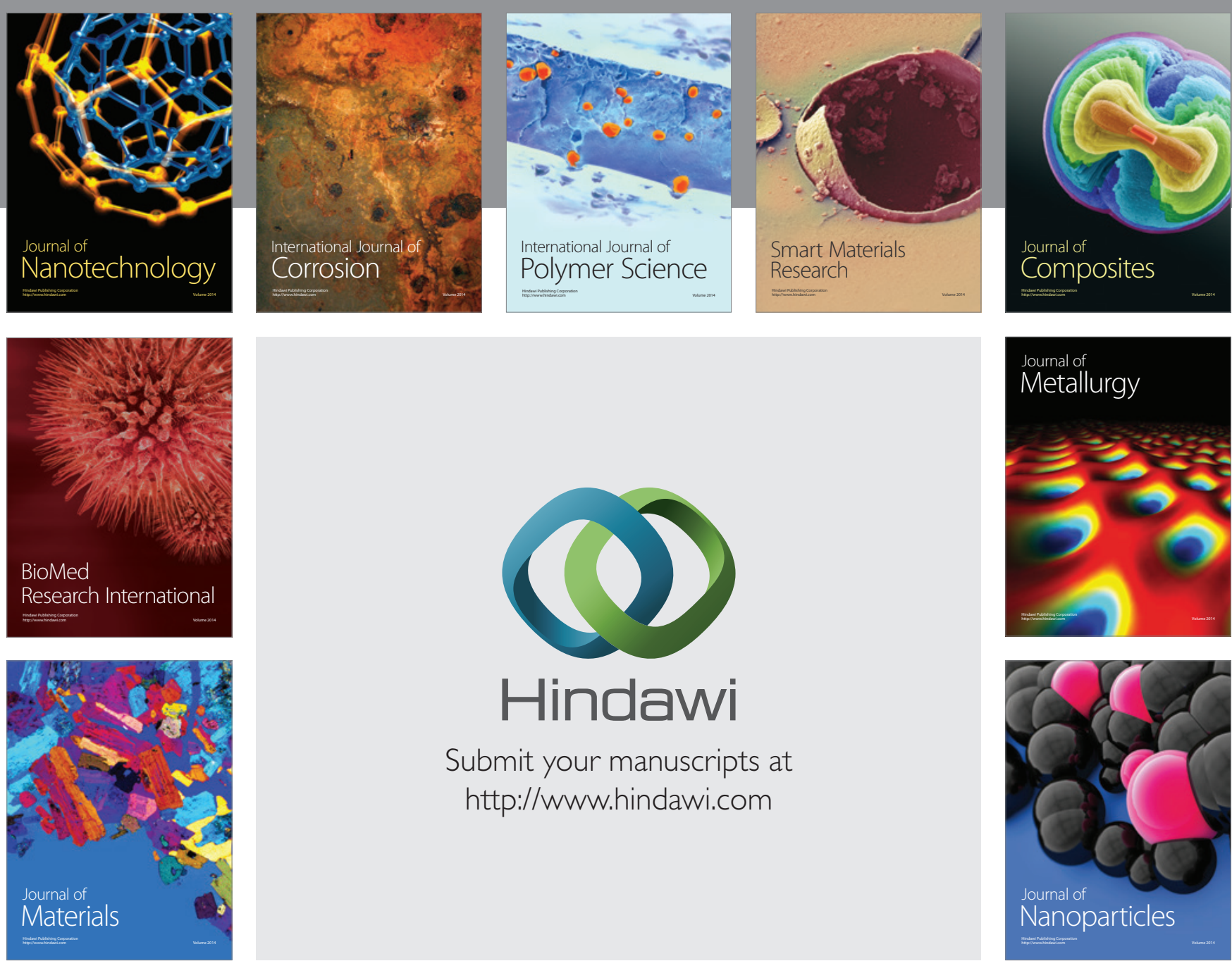

\section{Hindawi}

Submit your manuscripts at

http://www.hindawi.com

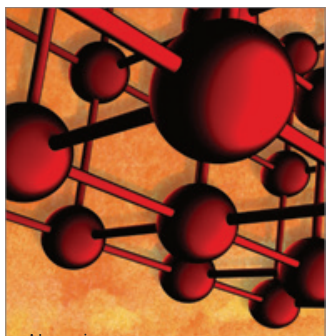

Materials Science and Engineering
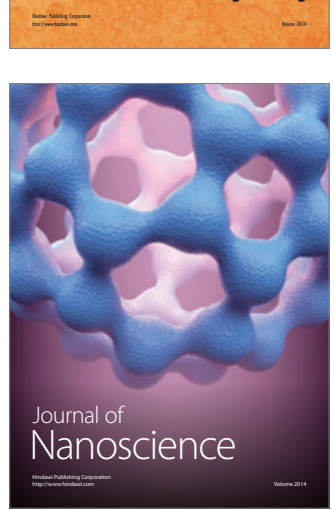
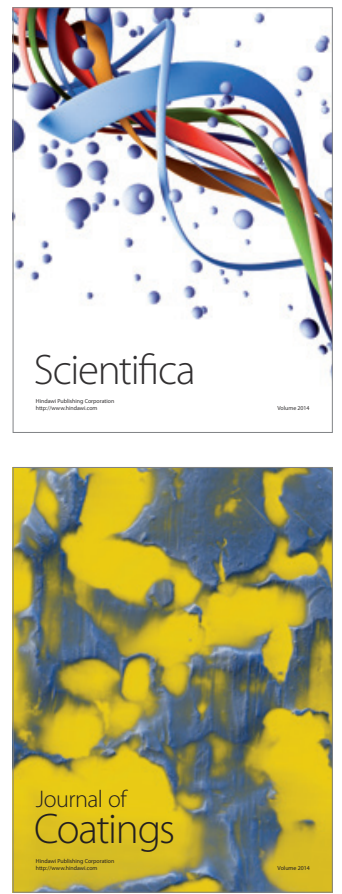
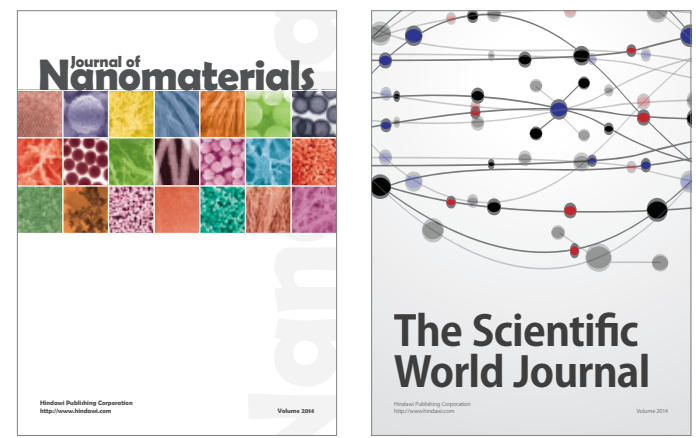

The Scientific World Journal
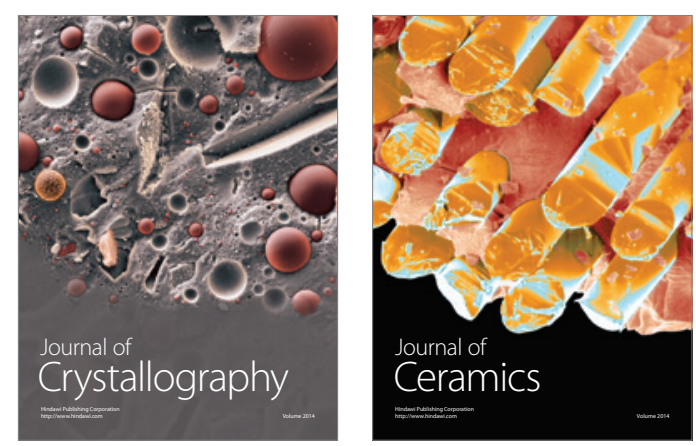
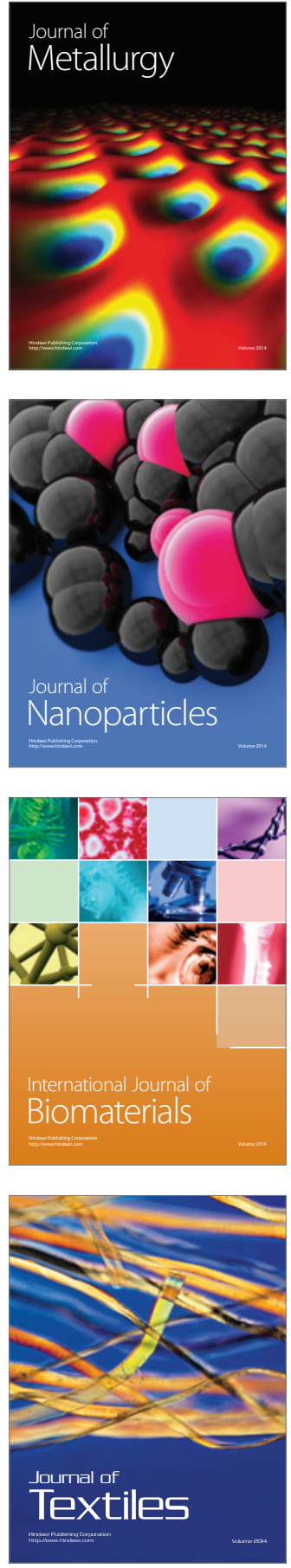